\title{
Low macular pigment optical density is associated with lower cognitive performance in a large, population-based sample of older adults
}

Feeney, J., Finucane, C., Savva, G. M., Cronin, H., Beatty, S., Nolan, J. M., \& Kenny, R. A., Low macular pigment optical density is associated with lower cognitive performance in a large, population-based sample of older adults.., Neurobiology of Aging, 34, 11, 2013, 2449 - 2456

\begin{abstract}
Macular pigment (MP) is comprised of the carotenoids lutein (L), zeaxanthin (Z), and meso-zeaxanthin (MZ), which selectively accumulate at the macula (central retina) of the eye and are neuroprotective. These carotenoids are also present in the brain, and evidence suggests a close correlation between retinal and brain concentrations. We investigated the relationship between MP and cognitive function in 4453 adults aged $\geq 50$ years as part of The Irish Longitudinal Study on Aging. Macular pigment optical density (MPOD) was determined using customized heterochromatic flicker photometry - a quick and noninvasive way of measuring the concentration of the pigment. Lower MPOD was associated with poorer performance on the mini-mental state examination $(\mathrm{p}=0.026)$ and on the Montreal cognitive assessment $(p=0.016)$. Individuals with lower MPOD also had poorer prospective memory $(\mathrm{p}=0.011)$, took longer time to complete a trail-making task ( $\mathrm{p}=0.003)$, and had slower and more variable reaction times on a choice reaction time task $(\mathrm{p}=0.000$ and 0.001$)$. These associations were only slightly attenuated following adjustment for physical and mental health. There was no significant association between MPOD and verbal fluency, word recall, visual reasoning, or picture memory. Overall, the findings support the theory that xanthophyll carotenoids impact on cognitive function, underscoring the need for exploration of novel, noninvasive biomarkers for cognitive vulnerability and preventive strategies.
\end{abstract}

Keywords

- Macular pigment optical density;

- Cognitive impairment;

- Aging

1. Introduction 
Identifying older people at risk of cognitive decline and developing interventions to prevent cognitive decline are public health priorities. Biomarkers are being sought with the aims of both early identification of those at risk and identification of possible pathways that could be targeted for preventive intervention. The eye is often regarded as a window to the brain, as the eye is relatively easy to noninvasively observe, and many pathologic changes in the eye reflect those throughout the central nervous system, including the brain.

The carotenoids lutein (L), zeaxanthin (Z), and meso-zeaxanthin (MZ) accumulate at the macula (to the exclusion of all other dietary carotenoids), where they are collectively known as macular pigment (MP). L and Z are entirely of dietary origin, whereas MZ is thought to derive (at least in part) from retinal $\mathrm{L}$ through a poorly understood bioconversion process (Bone et al., 1993), although its presence in the diet has not been comprehensively studied, and remains under investigation (Thurnham, 2007). MP is a powerful antioxidant (Khachik et al., 1997) and thus has a putative neuroprotective function in the retina. $\mathrm{L}$ and $\mathrm{Z}$, together with cryptoxanthin, comprise $\sim 70 \%$ of carotenoids in the frontal and occipital cortices (Craft et al., 2004), and there are several lines of evidence suggesting a neuroprotective effect of $\mathrm{L}$ and $\mathrm{Z}$ in the brain. $\mathrm{L}$ supplementation has been shown to improve verbal fluency in older women (Johnson et al., 2008), and plasma carotenoid levels have been associated with performance across a range of cognitive domains (Akbaraly et al., 2007) as well as being lower in individuals with mild cognitive impairment and Alzheimer's disease (AD) compared with normal controls (Rinaldi et al., 2003). Xanthophylls can attenuate the inflammatory effect of $A \beta$ on the vasculature, thus potentially reducing the risk of AD (Nakagawa et al., 2011). Furthermore, the concentration of $\mathrm{Z}$ in blood serum is inversely associated with the severity of white matter lesions in the brains of nondemented older adults (den Heijer et al., 2001). In a postmortem series, higher concentrations of $\mathrm{L}$ and $\mathrm{Z}$ in the brain were associated with better cognitive function at the time of death (Johnson et al., 2011). It has also been shown that the concentration of carotenoids comprising MP in the retina is highly correlated with carotenoid levels in the cerebellum and occipital cortex of macaque monkeys (Vishwanathan et al., 2013). This accruing body of evidence has led to the suggestion by Johnson (2012) that the density of MP may be used as a marker for the concentration of the constituent carotenoids in the cortex and cerebellum.

Whereas the measurement of serum carotenoids requires whole blood sampling with venepuncture, followed by expensive and challenging high-performance liquid chromatography, the density of carotenoids in the macula can be assessed rapidly and noninvasively using customized heterochromatic flicker photometry (cHFP) (Snodderly et al., 2004; Wooten et al., 1999). This technique exploits the optical properties of MP to quantify its density, yielding a measure termed "macular pigment optical density" (MPOD). MPOD therefore offers a noninvasive way to quickly assess carotenoid levels at the retina and therefore (potentially) the brain, which may help identify those at risk of potentially modifiable disease. 
Here, we investigate the relationship between MPOD and cognitive performance across a range of domains using data from wave 1 of The Irish Longitudinal study on Aging (TILDA), a large study of older adults representative of the population aged $\geq 50$ years in Ireland.

\section{Methods}

\subsection{Study design and participants}

TILDA is a nationally representative prospective cohort study of $>8000$ participants aged $\geq 50$ years which assesses the social, economic, and health circumstances of community-dwelling older adults in Ireland. Wave 1 of the study was completed in 2011, and wave 2 is currently underway. Three more waves of data collection have been planned. All household residents aged $\geq 50$ years and their spouses/partners (of any age) were eligible to participate in wave 1 . The study design and sampling methodology have been described previously (Kearney et al., 2011). The data presented herein were collected during wave 1 (baseline) of TILDA and thus are cross-sectional in nature.

All eligible participants initially completed an interview in their own home using computer-assisted personal interviewing (CAPI). Participants were left a selfcompletion questionnaire to complete in their own time and were also invited to have a comprehensive health assessment either in a dedicated health center or in their own home. All health assessments were carried out by trained research nurses. The overall response rate was $62 \%(n=8175)$. Of those, $85 \%$ returned the self-completion questionnaire $(n=6912)$ and $62 \%$ participated in a health center assessment $(n=5035)$. This study was approved by the Faculty of Health Sciences Research Ethics Committee of Trinity College, Dublin, and participants provided written informed consent before participation in the study.

\subsection{Cognitive assessment}

The TILDA health assessment included a comprehensive battery of cognitive tasks. Two measures of global cognition-the mini-mental state examination (MMSE) (Folstein et al., 1975) and the Montreal cognitive assessment (MoCA) (Nasreddine et al., 2005)-were administered as part of the health assessment. Verbal memory was assessed during the CAPI using a word-list learning and recall paradigm, in which participants were asked to recall 10 common, aurally presented words, both immediately and then again after a delay. The CAPI also included 2 prospective memory (PM) tasks, in which participants were informed about an action that they had to remember to carry out later in the interview. For the first PM (PM1) task, participants were instructed to remind the interviewer to record the time once a particular section of the interview had finished. For the second PM (PM2) task, participants were told that at some point later in the interview, they would be handed a piece of paper and to write their initials on the top, left-hand corner of this paper. Picture memory was assessed during the 
health assessment using the picture memory task from the Revised Cambridge Examination for Mental Disorders of the Elderly (CAMDEX-R) (Roth et al., 1999). Participants were shown pictures of 6 common objects and asked to name them. After a delay, they were asked to recall these objects from memory. This was followed by a forced-choice recognition task, whereby participants were presented with pictures containing an original object along with 2 similar objects and were asked to identify the original object in each case. Verbal fluency was assessed by asking participants to name as many animals as possible in 1 minute. Executive function was assessed using color trails tasks 1 and 2 (CTT1 and CTT2) (D'Elia et al., 1996) and the visual reasoning task from the CAMDEX-R (Roth et al., 1999). CTT1 is largely a test of visual scanning and sustained attention, whereas CTT2 is dependent on additional executive functions such as task switching (Rabelo et al., 2010). The performance variables of interest were the time taken to successfully complete CTT1 and the time taken to successfully complete CTT2, with shorter completion times indicative of better performance. In the visual reasoning task, participants were shown 3 patterned boxes with 1 blank box. They were asked to choose the correct pattern to fill the blank box from 6 options. Participants were given 6 of these problems to solve, and so the maximum possible score was 6 .

A computer-based choice reaction time (CRT) task was also administered during the health assessment as an index of mental processing speed. Participants were required to depress a central button until a stimulus appeared on-screen. Stimuli consisted of either the word "yes" or the word "no," and each time a stimulus appeared, participants were required to press the corresponding button. A return to the central button was necessary after each response for the next word to appear on-screen. The task variables of interest were the mean intraindividual RT and the standard deviation (SD) of individual RTs, the latter providing a measure of variability.

The sustained attention to response task (SART) (Robertson et al., 1997) was administered as a measure of attention. This task tests the ability of participants to self-sustain mindful, conscious processing of repetitive stimuli and to inhibit prepotent responses. The numbers 1 to 9 appear on a computer screen in consecutive order, and the participant is required to press the space bar in response to every number except the number 3 . The 1 to 9 sequence was repeated 23 times in total. Performance on the task was indexed by the number of errors of commission (pressing in response to the " 3 ") and errors of omission (failing to press in response to any of the numbers $1,2,4,5,6,7,8$, or 9).

\subsection{MPOD measurement}

MPOD was measured using the Macular Metrics Densitometer by cHFP (Providence, RI, USA). This device has been validated for the measurement of MPOD in older subjects (Snodderly et al., 2004; Stringham et al., 2008) and 
shown to be reliable (Gallaher et al., 2007; Snodderly et al., 2004). MPOD measurement by HFP has also been validated against the in vitro absorption spectrum of MP (Bone and Landrum, 2004; Hammond et al., 2005).

To measure MPOD, the participant views a flickering stimulus that alternates between a blue light (that is absorbed by MP) and a green light (not absorbed by MP), both at central (foveal) fixation where MP is at its highest density and again at a peripheral (parafoveal) point with optically negligible MP (Snodderly et al., 1984). At both fixation points, the radiance of the blue light is adjusted until the participant can no longer perceive the flicker, with the difference between the required radiance at the foveal and parafoveal points reflecting the participant's MPOD.

First, the examiner describes the task, using diagrams to ensure that the participant understands the task. The examiner selects the appropriate visual target to measure MPOD at the fovea $\left(0.5^{\circ}\right)$, and the participant places the eye with the best visual acuity (VA) at the viewing piece. The appropriate flicker frequency for the age of the participant is selected (Nolan et al., 2010). The examiner then sets the radiance to the lowest blue light intensity and presses a button that alters the blue/green radiance ratio until the participant reports that there is no flicker. This procedure is repeated twice to give a total of 3 measurements. The radiance dial is then set to the highest blue light intensity, and again the procedure is repeated 3 times as described previously. This gives 6 radiance values for the foveal measurement, 3 approaching from the lowest blue light intensity and 3 from the highest blue light intensity. The examiner then describes the parafoveal part of the measurement, again using diagrams. The appropriate visual target to measure MPOD at the parafovea ( $\sim 7^{\circ}$ eccentricity) is selected, and the procedure is repeated as mentioned previously to obtain 6 radiance values for the parafoveal measurement. These 12 values in total are then used to estimate MPOD for each participant by calculating the log ratio of the radiance values for the fovea $\left(0.5^{\circ}\right.$ eccentricity) to the reference point at $7^{\circ}$ eccentricity.

MPOD measurement was conducted as part of the health assessment and took place a maximum of 40 minutes after the cognitive testing. Because of the requirement for specialized equipment, MPOD measurement was carried out as part of the center-based health assessment only (i.e., was not carried out during in-home health assessments). Thus, 5035 participants were initially eligible for the measurement. Of these, 414 individuals were unable to complete the measurement because of an inability to understand the instructions. Corrected distance VA of at least 6/18 was deemed necessary for accurate MPOD measurement. Therefore, a further 95 participants with VA of worse than or equal to 0.5 on the logarithm of the Minimum Angle of Resolution (logMAR) scale were excluded from the measurement. Fifty-four individuals were unwilling to complete the measurement, and a technical issue with the densitometer 
prevented a further 19 individuals from completing the measurement. A total of 4453 individuals were therefore eligible for analysis (Fig. 1).

Fig. 1.

Population included in analysis.

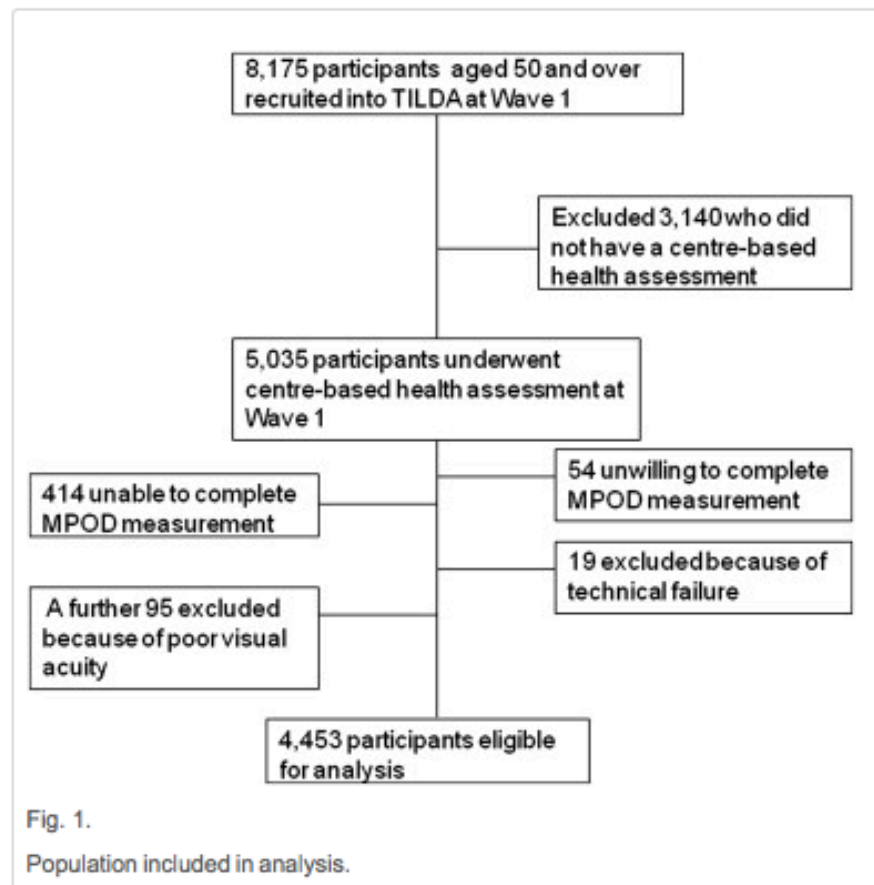

Figure options

\subsection{Potential confounding variables}

Both MPOD and cognition are known to be affected by health and health behaviors, and so available sociodemographic and health-related variables associated with cognitive function or MPOD were also included in the analysis. Demographic variables included age, sex, and highest level of education (none/primary, secondary, or tertiary/higher). Objective measures of health included blood pressure, total cholesterol, body mass index (BMI), and VA. Blood pressure was measured during the health assessment using a portable OMRON digital automatic blood pressure monitor, with the participant in a seated position. BMI was calculated for each participant from height and weight measurements that were carried out during the health assessment. Corrected VA was measured in both eyes using an Early Treatment Diabetic Retinopathy Study (ETDRS) logMAR chart at a test distance of $4 \mathrm{~m}$ using the subjects existing refractive correction (Nolan et al., 2011). Depressive symptoms were assessed during the CAPI using the 20-item Center for Epidemiologic Studies-Depression (CES-D) scale (Radloff, 1977). During the CAPI, participants were asked whether 
a doctor had ever told them that they suffered from any of a range of conditions including age-related macular degeneration (AMD) and diabetes. Participants were also asked about their smoking habits and were categorized into 3 groups based on their responses (nonsmoker, previous smoker, current smoker). The CAGE questionnaire (Mayfield et al., 1974) was included as a screen for problem drinking. Participants were defined as problem drinkers if they endorsed at least 2 out of the 4 items in the questionnaire (Bernadt et al., 1982). All the medications taken regularly by each participant were also recorded.

\subsection{Statistical analyses}

Stata (version 12.0) was used for all analyses. To account for the fact that the study response rate varied among different subgroups of the population, inverse probability weights were calculated for the main sample using the Quarterly National Household Survey (2010). Participation rates for the health center assessment also varied according to geographic location, health, education, age, smoking, and marital status, and so a specific "health center weight" was applied. All analyses were weighted and adjusted for the initial clustered sampling of TILDA participants.

\subsubsection{Descriptive statistics}

MPOD was recoded into quartiles for descriptive analysis. Initially, chi-squared tests were used to test the association between MPOD and categorical variables. Associations between MPOD and continuous demographic and health-related variables were explored using linear regression with Wald tests for global effects.

2.5.2. Regression analyses with cognitive performance as the outcome of interest

For the analysis of cognitive data, regression analyses were conducted with each cognitive test considered as an outcome and MPOD as the exposure variable. As the nature of the association between MPOD and cognitive outcomes varied depending on the outcome in question and was not always linear, analyses were carried out both with MPOD recoded into quartiles and with MPOD as a continuous variable. The results did not differ markedly between the 2 approaches, and so we present the results of the regression analysis using continuous MPOD as the exposure variable (Table 2), with differences in cognitive performance (MoCA and MMSE) by quartiles of MPOD displayed in Fig. 2. Linear, negative binomial or logistic regression models were applied as appropriate to the distribution of the cognitive variable. Mean and SD of CRT and time to complete CTT1 and CTT2 were log transformed before linear regression analysis to ensure normality of the residuals. Performance on each of the PM tasks reflected a binary outcome (success or failure), thus both PM1 and PM2 were analyzed using logistic regression. Errors of commission and omission on the SART were analyzed using negative binomial regression. MoCA and MMSE were analyzed using linear regression; however, as scores were discrete, 
bounded, and skewed, we conducted a sensitivity analysis calculating the number of errors made on each (the maximum possible score minus the actual score) and applying a negative binomial regression model. Results of the negative binomial regression analyses did not differ appreciably from the results of the linear regression analysis, and so the latter results are displayed for ease of interpretation.

Table 1. 


\begin{tabular}{|c|c|c|c|c|c|c|}
\hline \multicolumn{7}{|c|}{ Characteristics of participants by quartiles of MPOD } \\
\hline \multirow[b]{2}{*}{ Variable } & \multicolumn{4}{|c|}{ Quartiles of MPOD } & \multirow[b]{2}{*}{$p^{a}$} & \multirow[b]{2}{*}{$\begin{array}{l}\text { Missing MPOD } \\
(n=582)\end{array}$} \\
\hline & $\begin{array}{l}\text { Fourth } \\
\text { quartile }\end{array}$ & Third quartile & $\begin{array}{l}\text { Second } \\
\text { quartile }\end{array}$ & First quartile & & \\
\hline MPOD, mean (range) & $\begin{array}{l}0.42 \\
(0.31-1.01)\end{array}$ & $\begin{array}{l}0.25 \\
(0.19-0.31)\end{array}$ & $\begin{array}{l}0.14 \\
(0.08-0.19)\end{array}$ & $\begin{array}{l}0.02 \\
(0.00-0.08)\end{array}$ & & \\
\hline Age (years) & $62.8(9.6)$ & $62.4(8.8)$ & $62.2(8.6)$ & $62.3(8.5)$ & 0.742 & $69.5(9.1)$ \\
\hline Sex (male) $(\%)$ & 42.5 & 46.3 & 53.9 & 53.8 & 0.000 & 42.2 \\
\hline Tertiary education (\%) & 25.7 & 20.9 & 18.3 & 17.3 & 0.000 & 10.1 \\
\hline Current smoker $(\%)$ & 15.5 & 19.1 & 20.4 & 21.1 & 0.056 & 22.1 \\
\hline BMI, mean (SD) & $28.1(4.7)$ & $28.6(5.1)$ & $28.9(4.7)$ & $29.1(5.2)$ & 0.000 & $28.6(4.4)$ \\
\hline Cholesterol, mean (SD) & $5.1(1.1)$ & $5.1(1.1)$ & $5.1(1.1)$ & $5.1(1.1)$ & 0.925 & $4.9(0.9)$ \\
\hline VA, mean (SD) & $0.05(0.15)$ & $0.05(0.16)$ & $0.04(0.15)$ & $0.06(0.15)$ & 0.326 & $0.23(0.2)$ \\
\hline CES-D score, mean (SD) & $5.5(7.5)$ & $5.9(7.5)$ & $5.9(7.0)$ & $6.1(7.2)$ & 0.578 & $6.3(6.2)$ \\
\hline Antidepressant use (\%) & 5.5 & 6.8 & 5.7 & 7.4 & 0.368 & 8.9 \\
\hline Hypertension (\%) & 41.2 & 40.8 & 43.7 & 43.4 & 0.563 & 45.6 \\
\hline Diabetes (\%) & 6.2 & 7.0 & 7.5 & 7.8 & 0.655 & 9.0 \\
\hline $\operatorname{AMD}(\%)$ & 1.8 & 1.8 & 1.2 & 0.7 & 0.106 & 3.9 \\
\hline Problem drinking (\%) & 13.7 & 13.6 & 14.8 & 12.1 & 0.449 & 11.2 \\
\hline \multicolumn{7}{|l|}{ Cognition } \\
\hline MMSE score, mean (SD) & $28.6(1.7)$ & $28.7(1.6)$ & $28.5(1.7)$ & $28.2(1.8)$ & 0.000 & $26.9(2.4)$ \\
\hline MoCA score, mean (SD) & $25.2(3.3)$ & $25.1(3.2)$ & $24.9(3.4)$ & $24.6(3.1)$ & 0.000 & $21.9(3.7)$ \\
\hline PM1 (\% success) & 83.0 & 81.51 & 79.4 & 77.32 & 0.046 & 65.8 \\
\hline PM2 (\% success) & 87.6 & 86.2 & 87.5 & 83.5 & 0.105 & 74.1 \\
\hline $\begin{array}{l}\text { Immediate recall, mean } \\
\text { (SD) }\end{array}$ & $6.8(1.5)$ & $6.8(1.5)$ & $6.7(1.5)$ & $6.6(1.4)$ & 0.008 & $5.9(1.4)$ \\
\hline Delayed recall, mean (SD) & $6.2(2.3)$ & $6.1(2.3)$ & $6.0(2.2)$ & $5.9(2.2)$ & 0.059 & $5.0(1.9)$ \\
\hline Picture recall, mean (SD) & $3.3(1.1)$ & $3.2(1.1)$ & $3.2(1.1)$ & $3.2(1.1)$ & 0.130 & $2.9(1.1)$ \\
\hline $\begin{array}{l}\text { Picture recognition, mean } \\
\text { (SD) }\end{array}$ & $5.6(0.7)$ & $5.6(0.7)$ & $5.6(0.7)$ & $5.6(0.7)$ & 0.930 & $5.4(0.8)$ \\
\hline $\begin{array}{l}\text { CTT1 time (ms), mean } \\
\text { (SD) }\end{array}$ & $55.2(23.9)$ & $55.8(23.5)$ & $55.6(21.6)$ & $57.2(22.1)$ & 0.211 & $71.5(23.9)$ \\
\hline $\begin{array}{l}\text { CTT2 time (ms), mean } \\
\text { (SD) }\end{array}$ & $108.5(38.1)$ & $110.2(39.0)$ & $111.9(36.8)$ & $113.0(35.3)$ & 0.057 & $139.3(40.3)$ \\
\hline $\begin{array}{l}\text { Visual reasoning, mean } \\
\text { (SD) }\end{array}$ & $3.0(1.4)$ & $3.1(1.3)$ & $3.0(1.3)$ & $2.9(1.3)$ & 0.045 & $2.4(1.1)$ \\
\hline Verbal fluency, mean (SD) & $20.8(9.2)$ & $20.0(13.6)$ & $20.5(7.8)$ & $20.6(7.6)$ & 0.310 & $17.9(6.1)$ \\
\hline CRT (ms), mean (SD) & $796.2(210.6)$ & $784.7(180.2)$ & $798.9(173.0)$ & $824(201.8)$ & 0.000 & $938.1(209.1)$ \\
\hline $\begin{array}{l}\text { CRT variability }(\mathrm{ms}), \text { mean } \\
\text { (SD) }\end{array}$ & $288.1(338.9)$ & $287.6(313.3)$ & $296.6(307.4)$ & $329.2(332.7)$ & 0.012 & $468.7(347.6)$ \\
\hline $\begin{array}{l}\text { SART commission errors, } \\
\text { mean (SD) }\end{array}$ & $3.7(3.7)$ & $3.6(3.3)$ & $3.6(3.0)$ & $3.9(3.4)$ & 0.107 & $6.0(3.8)$ \\
\hline $\begin{array}{l}\text { SART omission errors, } \\
\text { mean (SD) }\end{array}$ & $6.7(8.0)$ & $6.3(7.3)$ & $6.9(7.9)$ & $7.5(8.0)$ & 0.089 & $13.9(9.8)$ \\
\hline
\end{tabular}

Key: AMD, age-related macular degeneration; BMI, body mass index; CES-D, Center for Epidemiologic Studies-Depression Scale; CRT, choice reaction time; CTT1 and CTT2 time, time to complete color trail tasks 1 and 2; MMSE, mini-mental state examination; MoCA, Montreal cognitive assessment; MPOD, macular pigment optical density; PM1 and PM2, first and second prospective memory tasks; SART, sustained attention to response task; SD, standard deviation; VA, visual acuity. 
Table 2.

Multivariate regression analyses of the association between cognitive test performance and MPOD (continuous variable) 


\begin{tabular}{|c|c|c|c|c|}
\hline \multirow[b]{3}{*}{ Cognitive variable } & \multicolumn{4}{|l|}{ MPOD } \\
\hline & \multicolumn{2}{|l|}{ Model $1^{a}$} & \multicolumn{2}{|l|}{ Model $2^{b}$} \\
\hline & $\beta / O R / \operatorname{RR}(95 \% \mathrm{Cl})$ & $p^{c}$ & $\beta /$ OR/IRR $(95 \% \mathrm{Cl})$ & $p^{c}$ \\
\hline \multicolumn{5}{|l|}{ Global cognition } \\
\hline MMSE & $\beta=0.48(0.06,0.90)$ & 0.026 & $0.42(-0.01,0.85)$ & 0.059 \\
\hline MoCA & $\beta=0.83(0.15,1.50)$ & 0.016 & $0.69(-0.00,1.36)$ & 0.053 \\
\hline \multicolumn{5}{|l|}{ Memory } \\
\hline PM1 success & $\mathrm{OR}=2.24(1.20,4.15)$ & 0.011 & $2.12(1.11,4.03)$ & 0.022 \\
\hline PM2 success & $\mathrm{OR}=1.61(0.80,3.25)$ & 0.181 & $1.56(0.77,3.16)$ & 0.221 \\
\hline Immediate recall & $\beta=0.22(-0.07,0.51)$ & 0.152 & $0.18(-0.12,0.48)$ & 0.232 \\
\hline Delayed recall & $\beta=0.27(-0.19,0.74)$ & 0.252 & $0.26(-0.22,0.74)$ & 0.282 \\
\hline Picture recall & $\beta=0.08(-0.14,0.31)$ & 0.465 & $0.08(-0.14,0.30)$ & 0.494 \\
\hline Picture recognition & $\beta=-0.00(-0.16,0.16)$ & 0.994 & $0.00(-0.15,0.16)$ & 0.949 \\
\hline \multicolumn{5}{|l|}{ Executive function } \\
\hline Log CTT1 time (ms) & $\beta=-0.06(-0.13,0.01)$ & 0.087 & $-0.04(-0.12,0.03)$ & 0.255 \\
\hline Log CTT2 time (ms) & $\beta=-0.09(-0.15,-0.03)$ & 0.003 & $-0.07(-0.13,-0.01)$ & 0.014 \\
\hline Visual reasoning & $\beta=0.19(-0.07,0.45)$ & 0.148 & $0.17(-0.09,0.44)$ & 0.192 \\
\hline Verbal fluency & $\beta=0.12(-1.20,1.44)$ & 0.857 & $0.00(-1.31,1.31)$ & 0.998 \\
\hline \multicolumn{5}{|l|}{ Processing speed } \\
\hline Log CRT mean (ms) & $\beta=-0.09(-0.13,-0.04)$ & 0.000 & $-0.08(-0.13,-0.03)$ & 0.001 \\
\hline Log CRT variability & $\beta=-0.33(-0.52,-0.13)$ & 0.001 & $-0.31(-0.52,-0.11)$ & 0.002 \\
\hline \multicolumn{5}{|l|}{ Sustained attention } \\
\hline SART commission errors & $\mathrm{IRR}=0.85(0.69,1.03)$ & 0.099 & $0.91(0.74,1.11)$ & 0.335 \\
\hline SART omission errors & IRR $=0.71(0.56,0.90)$ & 0.004 & $0.81(0.64,1.03)$ & 0.079 \\
\hline
\end{tabular}

Key: $\mathrm{Cl}$, confidence interval; CRT, choice reaction time; CTT1 and CTT2 time, time to complete color trail tasks 1 and 2 (log transformed); IRR, incidence rate ratio; MMSE, mini-mental state examination; MoCA, Montreal cognitive assessment; MPOD, macular pigment optical density; OR, odds ratio; PM1 and PM2, first and second prospective memory tasks; SART, sustained attention to response task.

a Adjusted for age, sex, and education.

b Adjusted for age, sex, education, smoking, hypertension, cholesterol, body mass index, visual acuity, diabetes, age-related macular degeneration, Center for Epidemiologic Studies-Depression scores, antidepressant use, and problem drinking.

C $\rho$ values that are significant at the $5 \%$ level are shown in bold.

Fig. 2.

Marginal mean (95\% confidence interval) performance on the Montreal Cognitive Assessment (MoCA) and Mini-Mental State Examination (MMSE) by quartile of macular pigment optical density (MPOD): (A) MoCA score and (B) MMSE score. ${ }^{*} \mathrm{p}<0.05$. 


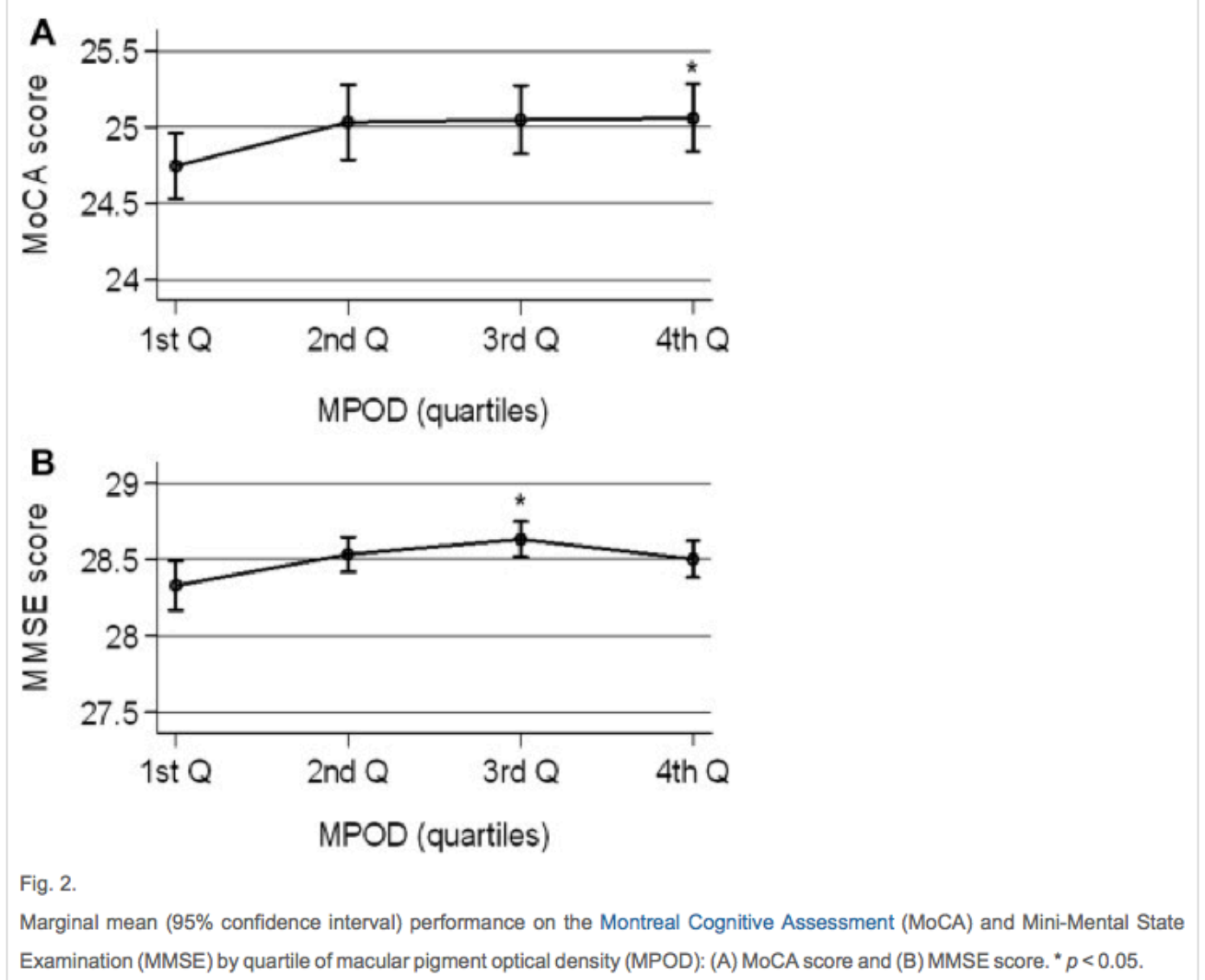

We adjusted our analyses for the potential confounding variables described in Section 2.4. Missing data on cognitive tasks and confounders was minimal, and so we included for each test only those participants who successfully completed the task. To ensure that any significant results identified were not simply a result of the large number of tests conducted and because of the high correlation among our outcome variables, we performed a multivariate analysis of variance (MANOVA) including each of the different cognitive tests as outcomes and MPOD (continuous) along with other covariates as predictors. MANOVA yields the $p$ value associated with the hypothesis that there is no association between the cognitive tests measured and MPOD, with a statistically significant $p$ value then indicating a likely association.

3. Results

MPOD ranged from 0 to 1.01 optical density units, with a mean of 0.20 and SD of 0.16. Mean (SD, range) of MPOD was $0.02(0.03,0.00-0.08)$ for the first quartile, $0.14(0.03,0.08-0.19)$ for the second quartile, $0.25(0.03,0.19-0.31)$ for the third quartile, and $0.42(0.11,0.31-1.01)$ for the fourth quartile (Table 1$)$.

3.1. Demographic characteristics and health-related variables 
Age did not differ greatly across quartiles of MPOD (mean $=62.4, \mathrm{SD}=9.0$ ). There was, however, a significant difference in the distribution of gender and education across the quartiles (Table 1). Individuals with higher MPOD were significantly less likely to be male $(\chi 2(3)=42.3, p=0.000)$ and more likely to have completed tertiary (or higher) education than those with lower MPOD $(\chi 2(3)=28.5, p=0.000)$. Those with lower MPOD had, on average, higher BMI than those with higher MPOD $(F(3,612)=6.53, p=0.000)$. There was also a significant bivariate association between MPOD and performance on many of the tests of cognitive function such that those with lower MPOD had poorer performance (MoCA: $F(3,611)=6.31, p=0.000$; MMSE: $F(3,612)=6.07, p=$ 0.000 ; immediate recall: $F(3,612)=3.98, p=0.008$; visual reasoning: $F(3,612)=$ 2.68, $\mathrm{p}=0.045$; PM1: $\mathrm{F}(3,612)=2.67, \mathrm{p}=0.046$; CRT mean: $\mathrm{F}(3,612)=6.06, \mathrm{p}=$ 0.000; CRT SD: $F(3,612)=3.67, p=0.012)$. There was no significant association between MPOD and verbal fluency $(\mathrm{F}(3,611)=1.20, \mathrm{p}=0.310)$, picture memory recall $(\mathrm{F}(3,612)=1.87, \mathrm{p}=0.13)$, recognition $(\mathrm{F}(3,612)=0.14, \mathrm{p}=0.93)$, delayed recall $(F(3,612)=2.49, p=0.059), P M 2(F(3,612)=2.05, p=0.105)$, or performance on the SART (omission errors: $F(3,611)=2.18, \mathrm{p}=0.089$; commission errors: $F(3,610)=2.04, p=0.107)$.

Individuals who did not undergo the MPOD measurement differed from those who completed the measurement in socioeconomic and health status (Table 1). Individuals who did not undergo the MPOD measurement were significantly older, less likely to be male and less likely to have completed tertiary education. They were significantly more likely to have AMD and to have lower cholesterol than those who completed the measurement. These individuals also performed worse on all tests of cognitive function. There was no difference between those with MPOD and those who did not undergo the measurement on any of the other health variables.

\subsection{Multivariate association between cognitive function and MPOD}

3.2.1. Model 1: adjusting for age, sex, and education

Lower MPOD remained associated with poorer performance on several tests of cognitive function, adjusting for age, sex, and education only (model 1, Table 2).

\subsubsection{Global cognitive function}

MMSE $(\beta=0.48,95 \%$ confidence interval $[\mathrm{CI}]=0.06,-0.90, \mathrm{p}=0.026)$ and MoCA $(\beta=0.83,95 \% \mathrm{CI}=0.15,1.50, \mathrm{p}=0.016)$ scores were significantly associated with MPOD.

\subsubsection{Memory}


Lower MPOD was significantly related to reduced probability of success on PM1 (odds ratio $=2.24,95 \% \mathrm{CI}=1.20,4.15, \mathrm{p}=0.011$ ). MPOD was not significantly associated with immediate word recall, delayed word recall, or picture memory ( Table 2).

\subsubsection{Executive function and processing speed}

There was no significant effect of MPOD on completion times for CTT1 (log transformed), although some evidence of a trend was apparent $(\beta=-0.06,95 \%$ $\mathrm{CI}=-0.13,0.01, \mathrm{p}=0.087)$. The association between MPOD and CTT2 completion times (log transformed) was statistically significant $(\beta=-0.09,95 \%$ $\mathrm{CI}=-0.15,-0.03, \mathrm{p}=0.003$ ), indicating that individuals with lower MPOD took longer time to successfully complete trail 2. Lower MPOD was significantly associated with slower RTs on the CRT task $(\beta=-0.09,95 \% \mathrm{CI}=-0.13,-0.04, \mathrm{p}$ $=0.000)$, and these individuals were also more variable $(\beta=-0.33,95 \% \mathrm{CI}=$ $-0.52,-0.13, p=0.001)$. There was evidence that individuals with lower MPOD had poorer sustained attention, with more errors of omission being committed by participants with lower pigment (incidence rate ratio $=0.71,95 \% \mathrm{CI}=0.56$, $0.90, p=0.004)$. There was no evidence of an association between MPOD and verbal fluency or visual reasoning ( Table 2).

\subsubsection{Model 2: full multivariate analysis}

Adjusting for cardiovascular health, self-reported AMD, depression, VA, antidepressant use, and problem drinking (model 2) did not substantially attenuate the relationships evident in model 1. Whereas associations between MPOD (continuous) and global cognition (MMSE and MoCA) and between MPOD and SART omission errors fell just outside conventional statistical significance, performance on PM1, CTT2, and CRT remained significantly associated with MPOD.

Fig. 2 displays the relationship between MPOD (quartiles) and performance on the MoCA and MMSE fully adjusting for confounders. Differences in performance between the fourth and first quartiles were significant at the 0.05 level for MoCA $(\beta=0.32,95 \% \mathrm{CI}=0.02,0.61, \mathrm{p}=0.034)$. Individuals in the third quartile had higher MMSE scores than those in the first quartile $(\beta=0.31,95 \% \mathrm{CI}=0.10,0.51$, $p=0.003$ ). The fact that the difference in MMSE scores between the first and third but not first and fourth quartiles was significant is likely related to a lack of variability and error in the measurement ( Hensel et al., 2007). Repeated followup assessments should give a more reliable picture of the relationship between MMSE performance and quartiles of MPOD.

\subsubsection{Multivariate analysis of variance}


MANOVA demonstrated a statistically significant association between cognitive performance (all tests) and MPOD after adjustment for age, sex, and educational attainment $(p=0.006)$ that remained significant after adjusting for all other covariates $(p=0.039)$. After removing the CRT task variables from the MANOVA model, the association between MPOD and cognitive performance remained statistically significant $(\mathrm{p}=0.015)$ adjusting for age, sex, and education and was close to statistically significant after adjusting for all other covariates ( $p=0.073)$, indicating that the observed differences are not driven solely by the strong association with the CRT task.

\section{Discussion}

In a large sample representative of community-dwelling adults aged $\geq 50$ years, those with lower MPOD performed significantly worse on tests of global cognitive function, executive function, memory, and processing speed. These associations largely persisted after adjusting for sociodemographic, health, and lifestyle factors. The cognitive differences between those with the highest and lowest levels of MPOD are substantial at the population level: those in the lowest quartile were $\sim 30 \%$ less likely to successfully complete a test of PM.

To our knowledge, this is the first study of this magnitude to explore the relationship between MPOD and cognitive function. Our findings are consistent with an association previously reported by Renzi et al. (2008), among MPOD, processing speed, and accuracy among 118 healthy older adults (Renzi et al., 2008). Several other pieces of evidence support a neuroprotective role of $L$ and $Z$ in the brain and hence MPOD as a marker for cognitive vulnerability. L and Z concentrations were positively related to premorbid cognitive function in the Georgia Centenarian Study (Johnson, 2012). Low serum concentrations of L and $\mathrm{Z}$ have been associated with AD and cognitive impairment (Kiko et al., 2012; Rinaldi et al., 2003) and also with AMD. AMD is the most common cause of blindness among the elderly in the western world and, interestingly, has been linked with an increased risk of dementia/cognitive impairment (Clemons et al., 2006; Klaver et al., 1999) and with a relative lack of MP (Beatty et al., 2001; Bone et al., 2001).

Measures of MPOD to assess tissue concentrations of $\mathrm{L}, \mathrm{Z}$, and MZ offer several advantages over serum carotenoid measurements because of the rapid and noninvasive technique used and because MP is less responsive to short-term dietary changes and so may better reflect long-term carotenoid status (Beatty et al., 2004; Nolan et al., 2007). The possible prognostic value of measuring MP in this regard is also premised on the known positive and significant relationship between concentrations of its constituent carotenoids in the retina and in the brain and on the observed association between MPOD and cognitive function. 
The association between MPOD and MoCA, MMSE, PM, and tests of executive control (e.g., CTT2) suggests a likely relationship between xanthophyll carotenoids and functional ability. Given that PM is vulnerable to early agerelated decline and that $\mathrm{PM}$ tests are sensitive to early-stage dementia (Huppert et al., 2000; Van den Berg et al., 2012), the possibility that simple dietary measures could delay the onset, or ameliorate the natural course, of dementia cannot be ignored.

The relationship of MPOD to executive function and PM implicates the frontal cortex, as this region has a well-established role in top-down cognitive control and mental flexibility, functions which impinge on everyday tasks such as planning and the execution of planned actions (e.g., Smith and Jonides, 1999; Stuss, 2011). Furthermore, as L and Z (together with cryptoxanthin) are the dominant carotenoids in the frontal cortex (Craft et al., 2004), a role for these compounds in the functionality of this area is possible and would also be consistent with vulnerability and consequential dysfunctionality when carotenoid levels are insufficient.

A strong relationship was observed between MPOD and CRT. CRT is a measure of processing speed, which has been proposed by some researchers to underpin age-related decline in many domains of cognition (Finkel et al., 2005, 2009; Salthouse, 1996, 2000; Verhaeghen and Salthouse, 1997). MP has also been shown to correlate with visual processing ability (Renzi and Hammond, 2010). However, additionally adjusting our results for CRT did not attenuate other observed relationships, suggesting that processing speed does not mediate the relationship between cognitive function and MPOD that we observed.

This study used a large, nationally representative population and employed a comprehensive cognitive battery. However, those living in long-term care institutions, those with severe cognitive impairments, and those who could not travel to a health center or could not complete the MPOD measurement were not included in the analysis. As those unable to complete the MPOD measure had poorer health and cognition than those who successfully underwent the measurement, their exclusion is likely to have attenuated, rather than accentuated, the relationships we observed and therefore should not be seen as a potential source of bias in this regard. The relative homogeneity of the TILDA sample (95\% Irish, Caucasian) could be viewed both as a strength and as a limitation of the study - decreasing MPOD variability attributable to differences in racial genetic profiles, while reducing comparability with more genetically heterogeneous populations, such as that of the United States.

The fact that the individuals who did not complete the MPOD measurement were significantly older than those who did may explain the absence of an association 
between age and MPOD. However, findings to date regarding the relationship between age and MPOD have been somewhat mixed (Berendschot and van Norren, 2005; Delori et al., 2001; Dietzel et al., 2011; Hammond and CarusoAvery, 2000; Renzi and Hammond, 2010); therefore, such a relationship cannot be assumed. Furthermore, although there is strong theoretical and empirical support for the protective effect of xanthophyll carotenoids on neural integrity, we sought to explore the possibility that cognition influences the measurement of MPOD by cHFP and whether such confounding (if any) would bias MPOD values toward zero. To address this issue, we conducted several sensitivity analyses, restricting the sample to only those with tertiary education and/or MoCA scores of $>26$, the latter reflecting individuals who would be likely to have the ability to successfully complete the MPOD measure. We found that the relationship between MPOD and cognition was still evident in these restricted samples, suggesting that the observed association did not arise from individuals lacking the cognitive capacity to understand and accurately follow the test instructions. The lack of blood carotenoid data is, however, a limitation of the study as knowing the serum carotenoid status of each individual would help to confirm the direction of the MPOD-cognition relationship.

Another possible limitation of the study rests on the lack of dietary information. MP is of dietary origin, which, in turn, is influenced by level of education and is likely to be linked to cognition. However, the published diet-MP relationship is modest (likely because of the difficulties in assessing carotenoid intake using food frequency questionnaires or food diaries) (Mares et al., 2006; Nolan et al., 2007), and adjusting for other factors important in determining the accumulation of carotenoids in the macula including body mass, lipid profile and smoking (Beatty et al., 2004) did not attenuate the relationships observed in the present study. We also were able to adjust for cardiovascular disease and hypertension, which are likely to mediate much of the link between poor diet and cognitive function.

In conclusion, MPOD is associated with global cognition, executive function, processing speed, and PM in a large, nationally representative study of older adults. This is a novel and important finding as it supports the thesis that MPOD measurement could offer a quick and noninvasive tool to assess this aspect of cognitive vulnerability in clinical practice (Johnson, 2012) and that dietary supplementation with L, Z, and MZ may offer a degree of neuroprotection in individuals with insufficient levels. However, given the borderline statistical significance of many of our findings, and null findings with respect to other cognitive variables, more work needs to be done to verify and refine our understanding of the observed relationships. In particular, as these results are cross-sectional comparisons, and thus causality is unknown, longitudinal analysis is required to determine whether MPOD predicts cognitive decline. Further, a technique of measuring MPOD that is independent of subject performance would represent a useful development for future studies if we are to facilitate the measurement of MPOD in as many individuals as possible. Future 
research will focus on the follow-up of these individuals through subsequent waves of TILDA to investigate any changes in cognitive function in relation to baseline MPOD.

Disclosure statement

The authors report no conflicts of interest.

Informed consent was obtained for all participants, and all protocols and procedures were approved by the institutional review board.

Acknowledgements

We would like to thank Professor Billy Wooten and Dr Robert Coen for their help and guidance. This work was supported by The Atlantic Philanthropies, Irish Life plc, and the Irish Government. 


\section{References}

Akbaraly, N.T., Faure, H., Gourlet, V., Favier, A., Berr, C., 2007. Plasma carotenoid levels and cognitive performance in an elderly population: results of the EVA study. J. Gerontol. A Biol. Sci. Med. Sci. 62, 308

Beatty, S., Murray, I.J., Henson, D.B., Carden, D., Koh, H., Boulton, M.E., 2001 . Macular pigment and risk for age-related macular degeneration in subjects from a Northern European population. Invest. Ophthalmol. Vis. Sci. 42, 439

Beatty, S., Nolan, J., Kavanagh, H., O

Donovanm, O., 2004. Macular pigment optical

density and its relationship with serum and dietary levels of lutein and zeax-

anthin. Arch. Biochem. Biophys. 430, 70

Berendschot, T.T., van Norren, D., 2005. On the age dependency of the macular pigment optical density. Exp. Eye Res. 81, 602

Bernadt, M.W., Mumford, J., Taylor, C., Smith, B., Murray, R.M., 1982. Comparison of questionnaire and laboratory tests in the detection of excessive drinking and alcoholism. Lancet 1, 325

Bone, R.A., Landrum, J.T., 2004. Heterochromatic

flicker photometry. Arch. Biochem.

Biophys. 430, 137

Bone, R.A., Landrum, J.T., Hime, G.W., Cains, A., Zamor, J., 1993. Stereochemistry of the human macular carotenoids. Invest. Ophthalmol. Vis. Sci. 34, 2033

Bone, R.A., Landrum, J.T., Mayne, S.T., Gomez, C.M., Tibor, S.E., Twaroska, E.E., 2001. Macular pigment in donor eyes with and without AMD: a case-control study.

Invest. Ophthalmol. Vis. Sci. 42, 235

Clemons, T.E., Rankin, M.W., McBee, W.L., 2006. Cognitive impairment in the AgeRelated Eye Disease Study: AREDS report no. 16. Arch. Ophthalmol. 124, 537

Craft, N.E., Haitema, T.B., Garnett, K.M., Fitch, K.A., Dorey, C.K., 2004. Carotenoid, tocopherol, and retinol concentrations in elderly human brain. J. Nutr. Health Aging 8, 156

D’Elia, L.F.S., Satz, P., Uchiyama, C.L., White, T., 1996. Color Trails Test: Professional Manual. Psychological Assessment Resources, Odessa, FL

Delori, F.C., Goger, D.G., Hammond, B.R., Snodderly, D.M., Burns, S.A., 2001 . Macular pigment density measured by autof luorescence spectrometry: comparison with reflectometry and heterochromatic

flicker photometry. J. Opt. Soc. Am. A Opt.

Image Sci. Vis. 18, 1212

den Heijer, T., Launer, L.J., de Groot, J.C., de Leeuw, F.E., Oudkerk, M., van Gijn, J., Hofman, A., Breteler, M.M., 2001. Serum carotenoids and cerebral white matter lesions: the Rotterdam scan study. J. Am. Geriatr. Soc. 49, 642

Dietzel, M., Zeimer, M., Heimes, B., Claes, B., Pauleikhoff, D., Hense, H.W., 2011.

Determinants of macular pigment optical density and its relation to age-related maculopathy: results from the Muenster Aging and Retina Study (MARS). Invest. Ophthalmol. Vis. Sci. 52, 3452

Finkel, D., Reynolds, C.A., McArdle, J.J., Hamagami, F., Pedersen, N.L., 2009. Genetic variance in processing speed drives variation in aging of spatial and memory abilities. Dev. Psychol. 45, 820

Finkel, D., Reynolds, C.A., McArdle, J.J., Pedersen, N.L., 2005. The longitudinal relationship between processing speed and cognitive ability: genetic and environmental influences. Behav. Genet. 35, 535

Folstein, M.F., Folstein, S.E., McHugh, P.R., 1975.

"Mini-mental state."A practicalmethod for grading the cognitive state of patients for the clinician. J. Psychiatr. Res. 12, 189

Gallaher, K.T., Mura, M., Todd, W.A., Harris, T.L., Kenyon, E., Harris, T., Johnson, K.C.,

Satter

field, S., Kritchevsky, S.B., Iannaccone, A., 2007. Estimation of macular pigment optical density in the elderly: test-retest variability and effect of optical

blur in pseudophakic subjects. Vision Res. 47, 1253

Hammond Jr., B.R., Caruso-Avery, M., 2000. Macular pigment optical density in a

Southwestern sample. Invest. Ophthalmol. Vis. Sci. 41, 1492

Hammond Jr., B.R., Wooten, B.R., Smollon, B., 2005. Assessment of the validity of in vivo methods of measuring human macular pigment optical density. Optom.

Vis. Sci. 82, 387

Hensel, A., Angermeyer, M.C., Riedel-Heller, S.G., 2007. Measuring cognitive change in older adults: reliable change indices for the Mini-Mental State Examination.

J. Neurol. Neurosurg. Psychiatry 78, 1298

Huppert, F.A., Johnson, T., Nickson, J., 2000. High prevalence of prospective memory

impairment in the elderly and in early-stage dementia:

findings from a

population-based study. Appl. Cogn. Psychol. 14, S63

Johnson, E.J., 2012. A possible role for lutein and zeaxanthin in cognitive function in the elderly. Am. J. Clin. Nutr. 96, 111S

Johnson, E.J., McDonald, K., Caldarella, S.M.,Chung, H.Y., Troen,A.M., Snodderly,D.M., 2008. Cognitive 
findings of an exploratory trial of docosahexaenoic acid andlutein supplementation in older women. Nutr. Neurosci. 11, 75 , E.J., Vishwanathan, R., Schalch, W., Poon, L., Wittwer, J., Johnson, M.A.,

Hausman, D., Davey, A., Green, R., Gearing, M., Woodard, J., Nelson, P.,

Miller, L.S., Scott, T.M., 2011. Brain levels of lutein (L) and zeaxanthin (Z) are

related to cognitive function in centenarians. FASEB J. 25, 975.21

Kearney, P.M., Cronin, H., O

Regan, C., Kamiya, Y., Savva, G.M., Whelan, B., Kenny, R.,

2011. Cohort profile: the Irish Longitudinal Study on Ageing. Int. J. Epidemiol. 40,

Khachik, F., Bernstein, P.S., Garland, D.L., 1997. Identi

fication of lutein and zeax-anthin oxidation products in human and monkey retinas. Invest. Ophthalmol.

Vis. Sci. 38, 1802

Kiko, T., Nakagawa, K., Tsuduki, T., Suzuki, T., Arai, H., Miyazawa, T., 2012. Signi

$\mathrm{f}$ icance of lutein in red blood cells of Alzheimer's disease patients. J. AlzheimersDis. 28, 593

J. Feeney et al. / Neurobiology of Aging 34 (2013) 2449

Klaver, C.C., Ott, A., Hofman, A., Assink, J.J., Breteler, M.M., de Jong, P.T., 1999. Is age-

related maculopathy associated with Alzheimer's Disease? The Rotterdam

Study. Am. J. Epidemiol. 150, 963

Mares, J.A., LaRowe, T.L., Snodderly, D.M., Moeller, S.M., Gruber, M.J., Klein, M.L.,

Wooten, B.R., Johnson, E.J., Chappell, R.J., 2006. Predictors of optical density of

lutein and zeaxanthin in retinas of older women in the Carotenoids in Age-

Related Eye Disease Study, an ancillary study of the Women's Health Initia-tive. Am. J. Clin. Nutr. 84, 1107

May

field, D., McLeod, G., Hall, P., 1974. The CAGE questionnaire: validation of a new

alcoholism screening instrument. Am. J. Psychiatry 131, 1121

Nakagawa, K., Kiko, T., Miyazawa, T., Sookwong, P., Tsuduki, T., Satoh, A., 2011.

Amyloid beta-induced erythrocytic damage and its attenuation by carotenoids.

FEBS Lett. 585, 1249

Nasreddine, Z.S., Phillips, N.A., Bedirian, V., Charbonneau, S., Whitehead, V., Collin, I.,

Cummings,J.L.,Chertkow,H.,2005.TheMontrealCognitiveAssessment,MoCA:a

briefscreeningtoolformildcognitiveimpairment.Am.Geriatr.Soc.53,695

Nolan, J.M., Kenny, R., O

Regan, C., Cronin, H., Loughman, J., Connolly, E.E., Kearney, P.,

Loane, E., Beatty, S., 2010. Macular pigment optical density in an ageing Irish

population:theIrishLongitudinalStudyonAgeing.OphthalmicRes.44,131

Nolan, J.M., Loughman, J., Akkali, M.C., Stack, J., Scanlon, G., Davison, P., Beatty, S.,

2011. The impact of macular pigment augmentation on visual performance in

normal subjects: COMPASS. Vision Res. 51, 459

Nolan, J.M., Stack, J., O

Connell, E., Beatty, S., 2007. The relationships between

macular pigment optical density and its constituent carotenoids in diet and

serum. Invest. Ophthalmol. Vis. Sci. 48, 571

Rabelo, I.S.A., Pacanaro, S.V., Rossetti, M.de.O., Leme, I.F.A.de.Sa., Castro, N.R.de.,

Güntert, C.M., Miotto, E.C., Lucia, M.C.S.de, 2010. Color Trails Test: a Brazilian

normative sample. Psychol. Neurosci. 3, 93

Radloff, L.S., 1977. The CES-D Scale: a self-report depression scale for the general

population. Appl. Psychol. Meas. 1, 385

Renzi,L.M.,Hammond,B.R.,2010.Therelationbetweenthemacularcarotenoids, lutein

and zeaxanthin, and temporal vision. Ophthalmic Physiol. Opt. 30, 351

Renzi, L.M., Iannacone, A., Johnson, E.J., Kritchevsky, S., 2008. The relation between

serum xanthophylls, fatty acids, macular pigment and cognitive function in the

Health ABC Study. FASEB J. 22, 877.5

Rinaldi, P., Polidori, M.C., Metastasio, A., Mariani, E., Mattioli, P., Cherubini, A.,

Catani, M., Cecchetti, R., Senin, U., Mecocci, P., 2003. Plasma antioxidants are

similarly depleted in mild cognitive impairment and in Alzheimer's disease.

Neurobiol. Aging 24, 915

Robertson, I.H., Manly, T., Andrade, J., Baddeley, B.T., Yiend, J., 1997.

formance correlates of everyday attentional failures in traumatic brain injured

and normal subjects. Neuropsychologia 35, 747

Roth, M., Huppert, F.A., Mountjoy, C.Q., Tym, E., 1999. The Revised Cambridge Examination for Mental Disorders of the Elderly, second ed. Cambridge University

Press, Cambridge

Salthouse, T.A., 1996. The processing-speed theory of adult age differences in cognition.

Psychol. Rev. 103, 403

Salthouse, T.A., 2000. Aging and measures of processing speed. Biol. Psychol. 54, 35

Smith, E.E., Jonides, J., 1999. Storage and executive processes in the frontal lobes.

Science 283, 1657

Snodderly, D.M., Auran, J.D., Delori, F.C., 1984. The macular pigment. II. Spatial

distribution in primate retinas. Invest. Ophthalmol. Vis. Sci. 25, 674

Snodderly, D.M., Mares, J.A., Wooten, B.R., Oxton, L., Gruber, M., Ficek, T., 2004.

Macular pigment measurement by heterochromatic

flicker photometry in older

subjects: the carotenoids and age-related eye disease study. Invest. Ophthalmol. 
Vis. Sci. 5, 531

Stringham, J.M., Hammond, B.R., Nolan, J.M., Wooten, B.R., Mammen, A., Smollon, W., Snodderly, D.M., 2008. The utility of using customized heterochromatic

flicker photometry (cHFP) to measure macular pigment in patients

with age-related macular degeneration. Exp. Eye Res. 87, 445

Stuss, D.T., 2011. Traumatic brain injury: relation to executive dysfunction and the frontal lobes. Curr. Opin. Neurol. 24, 584

Thurnham, D.I., 2007. Macular zeaxanthins and lutein

a review of dietary sources

and bioavailability and some relationships with macular pigment optical den-

sity and age related macular disease. Nutr. Res. Rev. 20, 163

Van den Berg, E., Kant, N., Postma, A., 2012. Remember to buy milk on the way

home! A meta-analytic review of prospective memory in mild cognitive

impairment and dementia. J. Int. Neuropsychol. Soc. 18, 706

Verhaeghen, P., Salthouse, T.A., 1997. Meta-analyses of age-cognition relations in

adulthood: estimates of linear and nonlinear age effects and structural models.

Psychol. Bull. 122, 231

Vishwanathan, R., Neuringer, M., Snodderly, D.M., Schalch, W., Johnson, E.J., 2013.

Macular lutein and zeaxanthin are related to brain lutein and zeaxanthin in

primates. Nutr. Neurosci 16, 21

Wooten, B.R., Hammond Jr., B.R., Land, R.I., Snodderly, D.M., 1999. A practical method for measuring macular pigment optical density. Invest. Ophthalmol.

Vis. Sci. 40,2481 